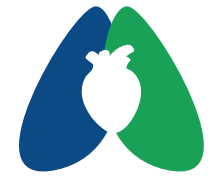

ASSOBRAFIR C I Ê N C I A

\section{Correlação entre a função respiratória e a obesidade em indivíduos candidatos à cirurgia bariátricaa}

\author{
Correlation between respiratory function and obesity in candidates \\ for bariatric surgery
}

Gabriel José Tarcisio Rodrigues ${ }^{1 *}$ (1); Danielle de Freitas Gonçalves² (D); Fabíola Pansani Maniglia² (D); Juliana Ribeiro Gouveia Reis ${ }^{1}$ (D).

\begin{abstract}
Resumo
Introdução: A obesidade é uma condição patológica caracterizada pelo excesso de gordura corporal, capaz de provocar prejuízos nos sistemas metabólico, respiratório e motor. Objetivo: Analisar se há correlação entre o Índice de Massa Corporal (IMC) e variáveis relacionadas a função inspiratória em indivíduos obesos candidatos a cirurgia bariátrica. Métodos: A amostra foi constituída por 54 indivíduos obesos em preparo para cirurgia bariátrica. Inicialmente foram coletadas informações pessoais para caracterização da amostra, em seguida foram avaliadas variáveis relacionadas a função inspiratória através do Powerbreathe ${ }^{\circledR} \mathrm{KH} 2$ com Respire-Link Medic Live Feedback Software (MIP pressão inspiratória máxima; PIF: pico de fluxo inspiratório, S-Index: Índice de Força). A análise estatística foi realizada através do programa SPSS-12; para verificar a existência de correlações, foi aplicado o Coeficiente de Correlação de Pearson. Resultados: Foram avaliados 54 obesos, sendo dez (18,52\%) homens e $44(81,48 \%)$ mulheres. Observou-se que os homens apresentaram

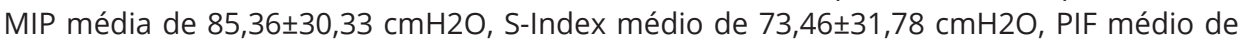

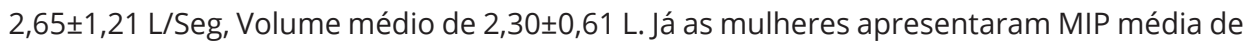

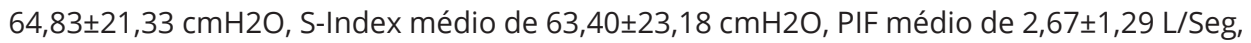

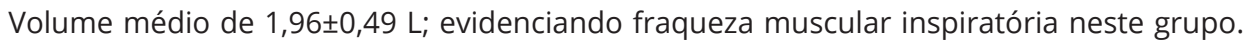
Além disso, verificou-se que não há correlação significativa entre as variáveis inspiratórias analisadas e o IMC dos participantes do estudo, evidenciado através da correlação entre IMC x MIP ( $r=-0,0607)$, IMC x S-Index ( $r=-0,0751)$, IMC x PIF $(r=-0,1335)$, IMC x VOLUME $(r=-0,0612)$. Conclusão: Verificou-se que não houve correlação significativa entre o IMC e as variáveis analisadas. Além disso, observou-se que mulheres obesas possuem fraqueza muscular inspiratória.
\end{abstract}

Palavras-chave: Obesidade; Mecânica Respiratória; Pressões Respiratórias Máximas; Índice de Massa Corporal.

\begin{abstract}
Background: Obesity is characterized by excess body fat, capable of causing damage to the metabolic, respiratory and motor systems. Aim: Analyze whether there's a correlation between body mass index (BMI) and variables related to inspiratory function in obese individuals who are candidates for bariatric surgery. Method: 54 obese individuals in preparation for bariatric surgery. Personal information and variables related to inspiratory function were evaluated using the Powerbreathe ${ }^{\circledR} \mathrm{KH} 2$ with Respire-Link Medic Live Feedback Software were collected. Statistical analysis was performed through the SPSS-12 program; to verify the existence of correlations, pearson's correlation coefficient was applied. Results: 54 individuals were evaluated, 10 (18.52\%) men and 44 (81.48\%) women. It was observed that men had an average MIP of $85.36 \pm 30.33 \mathrm{cmH} 2 \mathrm{O}$, an average SINDEX of $73.46 \pm 31.78 \mathrm{cmH} 2 \mathrm{O}$, an average FIP of $2.65 \pm 1.21 \mathrm{~L} / \mathrm{Sec}$, an average volume of 2, $30 \pm 0.61 \mathrm{~L}$. Women presented mean MIP of $64.83 \pm 21.33 \mathrm{cmH} 2 \mathrm{O}$, mean SINDEX of $63.40 \pm 23.18 \mathrm{cmH} 2 \mathrm{O}$, mean PIF of $2.67 \pm 1.29 \mathrm{~L} / \mathrm{Sec}$, Volume mean of $1.96 \pm 0.49 \mathrm{~L}$; showing inspiratory muscle weakness in this group. It was found that there isn't significant correlation between the inspiratory variables and the BMI, evidenced by the correlation between BMI x MIP $(-0.0607)$, BMI $\times$ SINDEX (-0.0751), BMI x PIF (-0.1335), BMI x VOLUME (-0.0612). Conclusion: It was found that there
\end{abstract}

Estudo realizado em: Instituto Pró-
CCATO, Patos de Minas, MG, Brasil.

Aprovação ética: Não se aplica.

Como citar: Rodrigues GJT, Gonçalves DF Maniglia FP, Reis JRG. Correlação entre a função respiratória e a obesidade em indivíduos candidatos à cirurgia bariátrica. RAFIR Ciênc. 2021;12:e43728. https://

Submissão em: Maio 19, 2021

Aceito em: Outubro 05, 2021

Gabriel José Tarcisio Rodrigues. E-mail:

fisioterapeutagabrieltarcisio@outlook.com 
was no significant correlation between $\mathrm{BMI}$ and the variables analyzed. It was observed that obese women have inspiratory muscle weakness.

Keywords: Obesity; Respiratory Mechanics; Physiotherapy; Maximal Respiratory Pressures; Body Mass Index.

\section{INTRODUÇÃO}

A obesidade é uma condição patológica caracterizada pelo excesso de gordura corporal acumulada', capaz de comprometer o estado de saúde dos indivíduos e consequentemente provocando prejuízos nos sistemas metabólicos, respiratório e motor ${ }^{2,3}$. Além disso, é um fator de risco determinante de patologias como dislipidemias, cardiopatias, valvulopatias, diabetes mellitus tipo II, dentre outras $2,4,5$.

Para diagnosticar um indivíduo com obesidade, a Organização Mundial de Saúde (OMS) ${ }^{1}$ determina que seja realizado o Índice de Massa Corporal (IMC), este parâmetro é estipulado a partir do peso corporal em quilogramas (Kg) e da estatura em metros ao quadrado $\left(\mathrm{m}^{2}\right)$. Indivíduos que apresentarem IMC igual ou superior a $30 \mathrm{~kg} / \mathrm{m}^{2}$ são considerados obesos. A etiologia da obesidade é determinada como multifatorial e de alta complexidade, envolvendo fatores biológicos, históricos, socioeconômicos, psicossociais e culturais ${ }^{6,7}$.

Dados epidemiológicos da Pesquisa Nacional de Saúde (PNS) de 2019, apontam que a taxa de pessoas com obesidade aumentou significativamente no Brasil nos últimos 17 anos, partindo de 12,2\% para 26,8\% de indivíduos diagnosticados com obesidade, ou seja, 96 milhões de brasileiros se encontram com sobrepeso. Além disso, conforme a média geral da PNS, apontou que a obesidade é mais observada em mulheres $(62,6 \%)$ do que em homens $(57,8 \%)^{8}$.

A obesidade é uma condição importante para complicações no sistema respiratório. O acúmulo de tecido adiposo na parede torácica, na região abdominal e sob a via aérea superior sobrecarregam o trabalho respiratório, promovendo alterações no sono e insuficiência respiratória hipercápnica. Além de limitar a mobilidade do diafragma e da parede torácica, consequentemente propiciando alterações na dinâmica respiratória e na complacência pulmonar $^{9}$.

Síndrome da Hipoventilação Alveolar da Obesidade e a Síndrome da Apneia do Sono podem estar relacionadas com o IMC superior ou igual a $30 \mathrm{~kg} / \mathrm{m}^{2}$, com a distribuição de tecido adiposo e com a idade. Além disso, observase comumente alterações nos volumes e capacidades pulmonares nos indivíduos obesos à medida em que há um aumento do IMC, consequente a sobrecarga da massa e da pressão impostas sobre o diafragma, além de uma maior resistência a entrada e saída de ar, promovida pelo estreitamento das vias aéreas ${ }^{10}$.

O conhecimento sobre a obesidade, suas comorbidades e sua ação sobre o sistema respiratório são extremamente importantes para os profissionais da saúde, principalmente para aqueles que lidam com este público e quando se trata de intervenções cirúrgicas ${ }^{11}$. Partindo deste pressuposto, objetivou-se com este estudo analisar se há correlação entre o Índice de Massa Corporal (IMC) e variáveis relacionadas a função inspiratória em indivíduos obesos candidatos a cirurgia bariátrica.

\section{MÉTODO}

Tratou-se de um estudo observacional do tipo transversal, realizado no Instituto Pró-Vida - CCATO em Patos de Minas/MG, com indivíduos obesos que realizaram acompanhamento fisioterapêutico pré-operatório para cirurgia bariátrica, entre setembro de 2020 e fevereiro de 2021. A coleta de dados iniciou-se após aprovação do projeto pelo Comitê de Ética e Pesquisa da Universidade de Franca (UNIFRAN), através do Parecer N 3.899 .280 e CAAE No: 28836820.0.0000.5395.

A amostra foi selecionada por conveniência, sendo selecionados 54 indivíduos obesos que estavam em habilitação pré cirúrgica no Instituto Pró-Vida - CCATO. Todos os indivíduos foram orientados sobre sua participação no estudo e aqueles que aceitaram participar assinaram o Termo de Consentimento Livre e Esclarecido (TCLE).

Foram incluídos neste estudo indivíduos com IMC igual ou superior a $30 \mathrm{~kg} / \mathrm{m}^{2}$, de ambos os sexos, com idade igual ou superior a 18 anos, que seriam submetidos a cirurgia bariátrica e que aceitaram participar do estudo após assinatura do TCLE. Como critério de exclusão foi definida a instabilidade hemodinâmica no momento da avaliação.

Após assinatura do TCLE, os pacientes foram submetidos a avaliação fisioterapêutica a fim de caracterizar a amostra da pesquisa, contendo dados como: nome, idade, peso, altura, IMC e data de nascimento. Em seguida foi realizada a avaliação dinâmica dos músculos inspiratórios através do aparelho Powerbreathe ${ }^{\circledR} \mathrm{KH} 2$ com Respire-Link Medic Live Feedback Software.

O Powerbreathe ${ }^{\circledR} \mathrm{KH} 2$ é um aparelho eletrônico portátil, não invasivo, que possibilita a avaliação da função pulmonar inspiratória e o treinamento muscular inspiratório com carga linear cuja adaptação é realizada de forma eletrônica ${ }^{12}$. Este instrumento possibilita a análise dinâmica da função e da musculatura inspiratória em diferentes volumes ou capacidades pulmonares ${ }^{13}$. Além disso, o dispositivo pode ser conectado ao software Breathelink, que proporciona um feedback visual, em tempo real, durante a sessão de avaliação ou de treinamento ${ }^{12}$.

Para realizar a avaliação inicialmente deve-se configurar o instrumento com informações pessoais de cada usuário, como idade, peso, altura, data de nascimento e sexo. Este equipamento possibilita a realização de três testes e a análise de variáveis respiratórias relacionadas a função inspiratória, as variáveis analisadas são a Pressão Inspiratória Máxima (MIP), o Pico de Fluxo Inspiratório (PIF) e o Índice de Força (S-Index) ${ }^{12}$. 
A medida da MIP é uma forma simples de avaliar e medir a força da musculatura inspiratória. Para realizar esta medida, o indivíduo deve ser orientado a realizar uma inspiração máxima, com uma das vias aéreas ocluídas, no caso a via aérea nasal ocluída; a partir do volume residual. O resultado é diretamente relacionado ao esforço realizado pelo usuário, para que seja eficaz é necessária que sejam repassadas instruções e que haja motivação encorajadora para a medição. O resultado da MIP exibido ao final do teste, corresponde à maior pressão média de 1 segundo alcançada durante a manobra (medido em $50 \mathrm{~Hz}$ ). Esta medida demonstra a pressão exercida pelos músculos inspiratórios associada a pressão de recolhimento elástico do sistema respiratório no volume residual. Resultado da MIP igual ou superior a $80 \mathrm{cmH} 2 \mathrm{O}$ exclui fraqueza da musculatura inspiratória ${ }^{12}$.

A medida do PIF, demonstra a capacidade dos músculos inspiratórios de se contraírem rapidamente e de vencer a resistência elástica imposta ao sistema respiratório. O resultado desta medida é diretamente relacionado a relação força-velocidade. Desta forma, o fluxo inspiratório pode apresentar redução em todos os volumes pulmonares em resposta a fraqueza da musculatura inspiratória ${ }^{12}$.

A medida do S-Index, é descrita como uma medida da força muscular inspiratória derivada do pico da medida do fluxo inspiratório, ou seja, é um valor previsto para MIP. O cálculo do índice de força é fundamentado em um padrão inspiratório e na relação força-velocidade da musculatura inspiratória. O resultado é classificado entre fraco a excelente, com base nos valores previstos que são calculados conforme as informações pessoais disponibilizadas no cadastro do usuário ${ }^{12}$.

Para análise estatística, incialmente os dados foram dispostos em uma planilha eletrônica no programa Excel 2019, no qual foram realizadas medidas de média e desvio padrão, em seguida foi realizada a análise através do programa SPSS-12. Com o objetivo de verificar a existência ou não de correlações, entre os valores de IMC e os valores de MIP, S-Index, PIF e Volume, foi aplicado o Coeficiente de Correlação de Pearson ${ }^{14}$, aos dados em questão. O nível de significância foi estabelecido em 0,05, em um teste bilateral, ou seja, para que os resultados das correlações fossem estatisticamente significantes, as probabilidades teriam que ser menores do que 0,05.

\section{RESULTADOS}

Participaram desta pesquisa 54 indivíduos obesos, candidatos à cirurgia bariátrica, sendo dez (18,52\%), do gênero masculino e $44(81,48 \%)$, do gênero feminino.

Em relação a caracterização da amostra, a Tabela 1 aponta a relação dos pacientes envolvidos na pesquisa e o estado civil, na qual observou-se que $36(66,6 \%)$ participantes declararam ser casados, 13 (24\%) declararam ser solteiros e $5(9,2 \%)$ declaram ser divorciados.
Já a Tabela 2 demonstra as 19 profissões que foram citadas pelos participantes, além daqueles que afirmaram estar desempregados (20,3\%) no momento da avaliação.

Na Tabela 3 e na Tabela 4, estão demonstrados os valores de médias e de desvios padrão, relativos às variáveis: idade, índice de massa corporal (IMC), pressão inspiratória máxima (MIP), pico da pressão inspiratória máxima (S-Index), pico de fluxo inspiratório (PIF) e volume pulmonar (volume), de acordo com o gênero e resultados totais.

A Tabela 5 demonstra a correlação entre o IMC e as variáveis inspiratórias. Ao analisar as probabilidades não foram encontradas correlações entre os valores das variáveis analisadas.

Tabela 1. Caracterização da amostra: estado civil.

\begin{tabular}{ccc}
\hline Estado Civil & $\mathrm{n}$ & $\%$ \\
\hline Casado (a) & 36 & $66,6 \%$ \\
Solteiro (a) & 13 & $24 \%$ \\
Divorciado (a) & 5 & $9,2 \%$ \\
\hline
\end{tabular}

Tabela 2. Caracterização da amostra: profissão.

\begin{tabular}{lcc}
\hline \multicolumn{1}{c}{ Profissão } & $\mathrm{n}$ & $\%$ \\
\hline Desempregado (a) & 11 & $20,3 \%$ \\
Empresário (a) & 10 & $18,5 \%$ \\
Confeiteiro (a) & 5 & $9,2 \%$ \\
Vendedor (a) & 4 & $7,4 \%$ \\
Autônomo (a) & 3 & $5,5 \%$ \\
Advogado (a) & 2 & $3,7 \%$ \\
Auxiliar Administrativo & 2 & $3,7 \%$ \\
Balconista/Recepcionista & 2 & $3,7 \%$ \\
Dentista & 2 & $3,7 \%$ \\
Gerente Bancário & 2 & $3,7 \%$ \\
Professor (a) & 2 & $3,7 \%$ \\
Outras & 0 & $15 \%$ \\
\hline
\end{tabular}

Tabela 3. Médias (X) e Desvios Padrão (DP), relativos às variáveis, idade e IMC.

\begin{tabular}{cccc}
\hline Variáveis & Masculino & Feminino & Total \\
\hline & $\mathrm{X} \pm \mathrm{DP}$ & $\mathrm{X} \pm \mathrm{DP}$ & $\mathrm{X} \pm \mathrm{DP}$ \\
Idade (anos) & $37 \pm 7$ & $36 \pm 10$ & $36 \pm 9$ \\
$\mathrm{IMC}\left(\mathrm{kg} / \mathrm{m}^{2}\right)$ & $43,41 \pm 4,15$ & $40,98 \pm 4,86$ & $41,43 \pm 4,80$ \\
\hline
\end{tabular}


Tabela 4. Médias (X) e Desvios Padrão (DP), relativos às variáveis, MIP, SINDEX, PIF e volume pulmonar, de acordo com o gênero e resultados totais.

\begin{tabular}{|c|c|c|c|}
\hline \multirow{2}{*}{ Variáveis } & Masculino & Feminino & Total \\
\hline & $\mathrm{X} \pm \mathrm{DP}$ & $X \pm D P$ & $X \pm D P$ \\
\hline $\mathrm{MIP}(\mathrm{cmH} 2 \mathrm{O})$ & $85,36 \pm 30,33$ & $64,83 \pm 21,33$ & $68,63 \pm 24,29$ \\
\hline $\begin{array}{l}\text { S-Index } \\
(\mathrm{cmH2O})\end{array}$ & $73,46 \pm 31,78$ & $63,40 \pm 23,18$ & $65,26 \pm 24,96$ \\
\hline PIF (L/seg) & $2,65 \pm 1.21$ & $2,67 \pm 1,29$ & $2,67 \pm 1,27$ \\
\hline Volume (L) & $2,30 \pm 0,61$ & $1,96 \pm 0,49$ & $2,02 \pm 0,53$ \\
\hline
\end{tabular}

MIP pressão inspiratória máxima; PIF: pico de fluxo inspiratório, S-Index: Índice de Força

Tabela 5. Valores de $r$ e das probabilidades ( $p$-valor) a eles associadas referente aos valores de IMC e aos valores de MIP, S-Index, PIF e Volume.

\begin{tabular}{lcc}
\hline $\begin{array}{l}\text { Variáveis } \\
\text { Analisadas }\end{array}$ & Valores de $r$ & p-valor \\
\hline IMC x MIP & $-0,0607$ & 0,663 \\
IMC x S-Index & $-0,0751$ & 0,589 \\
IMC x PIF & $-0,1335$ & 0,336 \\
IMC x Volume & $-0,0612$ & 0,660
\end{tabular}

MIP pressão inspiratória máxima; PIF: pico de fluxo inspiratório, S-Index: Índice de Força

\section{DISCUSSÃO}

Através da análise da correlação, observou-se que as variáveis IMC x MIP apresentaram índice de correlação de -0,607, IMC x S - Index índice de correlação de -0,751, IMC x PIF índice de correlação de -0,1335 e IMC x Volume índice de correlação de -0,0612; não demonstrando correlação estatisticamente significativa entre as variáveis analisadas. Todavia, observou-se que as mulheres da amostra apresentaram menores valores de força muscular inspiratória (MIP) $(64,83 \pm 21,33 \mathrm{cmH} 20)$ indicando fraqueza desta musculatura ( $<80 \mathrm{cmH} 2 \mathrm{O}$ ). Já a amostra masculina apresentou uma média maior que $80 \mathrm{cmH} 2 \mathrm{O}$, não indicando fraqueza da musculatura inspiratória.

Segundo Junior et al. ${ }^{15}$, em seu estudo realizado com 21 mulheres com idade média de 49 anos, sendo $14 \mathrm{com}$ obesidade mórbida e IMC médio de $55,03 \mathrm{Kg} / \mathrm{m}^{2}$, e 7 mulheres com IMC médio de $24,97 \mathrm{~kg} / \mathrm{m}^{2}$, descrito como normal. Os autores observaram que as mulheres com obesidade mórbida apresentaram redução significativa da força muscular inspiratória, principalmente quando comparadas ao grupo de mulheres com IMC normal que apresentavam força muscular inspiratória dentro da normalidade.

Sonehara et al. ${ }^{16}$, também encontraram resultados semelhantes aos apresentados neste estudo. Os pesquisadores avaliaram 20 mulheres obesas e sedentárias, com idade média de 48,8 \pm 13,7 e IMC médio de $34,54 \pm 3,85 \mathrm{~kg} / \mathrm{m}^{2}$, no qual observaram fraqueza da musculatura inspiratória, obtendo uma média na medida da pressão inspiratória máxima de 69,75 $\pm 21,37$ cmH2O.

Acredita-se que a redução da força muscular inspiratória em obesos esteja relacionada com o excesso de massa corporal sobre os músculos inspiratórios ${ }^{17}$. Além disso, as restrições impostas ao sistema respiratório pela caixa torácica e pelo excesso de gordura no abdome, altera a mobilidade do diafragma e toda sua dinâmica ${ }^{18}$. Outra possível explicação, é a relação com a redução da complacência torácica observada em obesos, uma vez que o excesso de tecido adiposo sobre o tórax e o abdome favorecem uma compressão da caixa torácica com consequente diminuição da complacência e alteração da mecânica ${ }^{19}$.

Em relação a correlação entre os dados, e corroborando com o resultado do presente estudo, Soares et al. ${ }^{20}$, em seu estudo realizado com 13 mulheres obesas com idade média de $44 \pm 11$ anos e IMC médio de $43 \pm 5 \mathrm{~kg} / \mathrm{m}^{2}$, os autores observaram que não houve correlação significativa entre o IMC e a MIP das participantes do estudo, todavia as medidas de pressão inspiratória máxima foram realizadas através de manovacuometria.

Conforme Sarykaya et al. ${ }^{21}$, também encontraram resultados aproximados aqueles observados no presente estudo, ao analisarem 51 indivíduos obesos com idade média de $42,78 \pm 10,8$ anos os estudiosos observaram não haver correlação significativa entre o IMC e a força muscular inspiratória dos participantes, determinada pela PI Máxima; esta medida também foi realizada através de manovacuometria. Costa et al. ${ }^{22}$, demonstram em seu estudo, que as variáveis antropométricas não apresentam correlação significativa com a pressão inspiratória máxima.

Segundo o estudo de Melo et al. ${ }^{23}$, ao analisarem a correlação entre o IMC e a pressão inspiratória máxima e a pressão expiratória máxima em 140 indivíduos obesos com idade média de 36,4 $\pm 11,2$ anos e IMC médio de $39,91 \pm 10,57 \mathrm{~kg} / \mathrm{m}^{2}$, os pesquisadores observaram que aqueles com IMC $\leq 45,0 \mathrm{~kg} / \mathrm{m}$ apresentavam pouca alteração das variáveis respiratórias, enquanto aqueles com $I M C \geq 45,0 \mathrm{~kg} / \mathrm{m}^{2}$ demonstravam alterações significativas da força muscular respiratória, intensificando-se naqueles com IMC de 50,9 kg/m².

Acredita-se que esses resultados estejam relacionados a maior quantidade de fibras musculares do tipo II e a menor quantidade de fibras musculares do tipo I encontradas em indivíduos obesos, como resposta à sobrecarga crônica determinada pelo excesso de tecido adiposo, desta forma o predomínio de fibras musculares do tipo II possibilita maior capacidade de produzir ou manter a força muscular respiratória ${ }^{24,25}$.

Outro fator apontado por pesquisadores que pode estar relacionado a pressão inspiratória máxima em indivíduos obesos, é a presença da síndrome de obesidade-hipoventilação, observada em diversos indivíduos. As disfunções consequentes desta síndrome 
podem estar relacionadas a redução da pressão inspiratória máxima nesta população ${ }^{26}$. Todavia, todos os participantes do presente estudo foram questionados sobre a presença de síndromes ventilatórias, não sendo relatada por nenhum deles.

Estudiosos apontam que as pressões respiratórias máximas podem se apresentar normal em indivíduos com obesidade, que não apresentam comorbidades relacionadas, até mesmo naqueles com obesidade mórbida ${ }^{27,28,29}$. Segundo Laghi e Tobin ${ }^{29}$ os indivíduos obesos suprem as pressões respiratórias máximas, aumentando o trabalho do sistema respiratório e a pressão diafragmática, associando a maior contribuição da caixa torácica no movimento respiratório, realizando respirações rápidas e superficiais, justificando a normalidade destas pressões.

Contradizendo estas citações, Poulain et al..$^{30}$ apontam que as pressões respiratórias máximas podem estar reduzidas em indivíduos com alto IMC, principalmente a pressão inspiratória máxima, sendo consequência da redução da complacência pulmonar e dos volumes e capacidades pulmonares. Segundo Ray et al..$^{31}$ e Koenig ${ }^{19}$ a redução das pressões respiratórias máximas pode estar relacionada a diminuição na mobilidade do diafragma, devido às restrições sobre a caixa torácica, causadas pelo excesso de tecido adiposo.

Conforme o estudo de Castello et al. ${ }^{17}$, no qual os autores analisaram a pressão inspiratória máxima e a pressão expiratória máxima de 12 mulheres com obesidade mórbida com idade média de $26 \pm 5$ anos e IMC de $47 \pm 6 \mathrm{~kg} / \mathrm{m}$ e 12 mulheres eutróficas com idade média de $27 \pm 4$ anos e IMC de $24 \pm 2 \mathrm{~kg} / \mathrm{m} 2$, os pesquisadores constataram que as pressões respiratórias máximas apresentaram redução significativa nas mulheres com obesidade mórbida, quando comparadas às eutróficas.

Corroborando com os dados de Castello et al. ${ }^{17}$, Junior et al. ${ }^{15}$, em seu estudo realizado com 21 mulheres, sendo 14 obesas mórbidas com idade média de $49,6 \pm 13,43$ anos e IMC de 55,03 $\pm 6,94 \mathrm{~kg} / \mathrm{m}$ e 7 mulheres eutróficas com idade média de 49,71 $\pm 4,92$ anos e IMC de $24,97 \pm 2,95 \mathrm{~kg} / \mathrm{m} 2$, os autores observaram que as mulheres obesas mórbidas apresentaram pressões respiratórias máximas reduzidas, quando comparadas ao grupo de mulheres eutróficas.

Não foram encontrados estudos na literatura que falassem da correlação do IMC com o volume pulmonar avaliado no aparelho Powerbreathe $\mathrm{KH} 2$, todavia Mafortt et al. ${ }^{32}$, afirma que em indivíduos obesos comumente observa redução do volume pulmonar, devido ao aumento da resistência gerada sobre as vias aéreas. Ainda segundo o pesquisador, as explicações não são totalmente conhecidas, entretanto acredita-se que esta redução esteja relacionada a remodelação das estruturas das vias aéreas, consequente a maior exposição a adipocinas pró-inflamatórias ou pela maior deposição de lipídios.

As principais limitações observadas no presente estudo, foram a heterogeneidade de amostra entre os gêneros e a ausência de cálculo amostral, além da falta de estudos na literatura que abordem a avaliação das variáveis respiratórias analisadas através do Powerbretahe $\mathrm{KH} 2$ e as correlacione com o IMC de indivíduos obesos, comprometendo a discussão deste estudo. Observou-se um maior número de estudos que utilizaram tal equipamento para o treinamento de musculatura inspiratória. Todavia, espera-se que a partir deste estudo, outros sejam realizados a fim de comprovar os resultados encontrados.

\section{CONCLUSÃO}

Através dos resultados observados neste estudo, conclui-se que não há correlação significativa entre as variáveis, MIP, PIF, S-Index e Volume, relacionadas ao sistema respiratório (inspiratório) e o Índice de Massa Corporal de indivíduos obesos candidatos a cirurgia bariátrica. Os resultados encontrados são preliminares, sendo a amostra selecionada referente a outro estudo em questão, sugere-se que novos estudos sejam reproduzidos a fim de corroborar com os dados deste estudo.

Devido à escassez de estudos na literatura que expõem o tema discutido no presente estudo, sugere-se também que novos trabalhos que abordem as variáveis MIP, PIF, S-Index e Volume correlacionando-os com o IMC sejam realizados com a população de indivíduos brasileiros obesos, a fim de confrontá-los com a literatura e compreender melhor o princípio das alterações provocadas pela obesidade no sistema respiratório. Ademais, espera-se que este estudo contribua com a realização de novas pesquisas sobre esse assunto.

\section{FONTE DE FINANCIAMENTO}

Nada a declarar.

\section{CONFLITO DE INTERESSES}

Nada a declarar.

\section{REFERÊNCIAS}

1. WHO: Word Health Organization. Obesity: preventing and managing the global epidemic. Report of a WHO Consulation on Obesity. Geneva: WHO, 1998.

2. Monteiro CA, Conde WL. A tendência secular da obesidade segundo estratos sociais: nordeste e sudeste do Brasil, 19751989-1997. Arq Bras Endocrinol Metabol. 1999;43(3):186-94. http://dx.doi.org/10.1590/S0004-27301999000300004.

3. Pereira LO, Francischi RP, Lancha HAJr. Obesidade: hábitos nutricionais, sedentarismo e resistência à insulina. Arq Bras Endocrinol Metabol. 2003;47(2):111-27.

4. Kac G, Velásquez-Meléndez G. A transição nutricional e a epidemiologia da obesidade na América Latina. Cad Saúde Pública. 2003;19(1):4-5. http://dx.doi.org/10.1590/S0102$311 \times 2003000700001$. 
5. Anjos LA. Obesidade e saúde pública. Rio de Janeiro: Fiocruz; 2006.

6. Brasil: Ministério da Saúde: Secretaria de Atenção à Saúde. Cadernos de atenção básica: obesidade. Brasília: Ministério da Saúde; 2006.

7. Stunkard AJ. Factores determinantes de la obesidad: opinión actual. In: Peña M, Bascallao J, editores. La obesidad en la pobreza: un nuevo reto para la salud pública. Washington, D.C.: OPS/OMS; 2000.

8. ABESO: Associação Brasileira para o Estudo da Obesidade e da Síndrome Metabólica [Internet]. Os últimos números da obesidade no Brasil. São Paulo: ABESO; 2020. [citado em 2021 Fev 20]. Disponível em: https://abeso.org.br/osultimos-numeros-da-obesidade-no-brasil/

9. Machado MGR, Ribeiro S. Fisioterapia respiratória no pré e pós-operatório de cirurgias abdominais. In: Machado, MGR., editors. Bases da fisioterapia respiratória. Rio de Janeiro: Guanabara Koogan, 2019. p. 359-365.

10. Araújo IFLA, Cabral VP. Fisioterapia e a cirurgia bariátrica. In: Pereira A, Freire CC, Godoy EP, Viegas F, Marche JCD, Zanella MT, editors. Cirurgia bariátrica e metabólica: abordagem multiprofissional. Rio de Janeiro: Rubio, 2019. p. 139-149.

11. Araújo OC, Espada EB, Costa FMA, Vigiato JA, Carmona MJC, Otoch JP, et al. Impacto da obesidade grau I na mecânica respiratória durante cirurgia videolaparoscópica: estudo longitudinal prospectivo. Rev Bras Anestesiol. 2020;20(2):906. http://dx.doi.org/10.1016/j.bjan.2019.12.001.

12. PowerBreathe Internacional LTD. POWERbreathe $®$ K Series: user manual English. United Kingdom: E \& OE, 2015. 44 p.

13. Lima PFP. Função cardíaca: predição de carga de treinamento muscular inspiratório e correlação com dados pneumofuncionais de indivíduos com insuficiência cardíaca. [Dissertação de Mestrado em Fisioterapia]. Recife: Universidade Federal de Pernambuco; 2016.

14. Graner EA. Estatística. São Paulo: Melhoramentos, 1966. $184 \mathrm{p}$.

15. Sant Anna M Jr, Oliveira JEP, Carneiro JRI, Guimarães FS, Torres DFM, Moreno AM, et al. Força muscular respiratória de mulheres obesas mórbidas e eutróficas. Fisioter Pesqui. 2011;18(2):122-6. http://dx.doi.org/10.1590/S180929502011000200004.

16. Sonehara E, Cruz MSL, Fernandes PR, Policarpo F, Fernandes J Fo. Efeitos de um programa de reabilitação pulmonar sobre mecânica respiratória e qualidade de vida de mulheres obesas. Fisioter Mov. 2011;24(1):13-21. http://dx.doi. org/10.1590/S0103-51502011000100002.

17. Castello V, Simões RP, Bassi D, Mendes RG, Borgi-Silva A. Força muscular respiratória é marcantemente reduzida em mulheres obesas mórbidas. Arq Méd ABC. 2007;32(2):74-7.

18. Stirbulov R. Repercussões respiratórias da obesidade. J Bras Pneumol. 2007;33(1):vii-viii. http://dx.doi.org/10.1590/ S1806-37132007000100003.

19. Koenig SM. Pulmonary complications of obesity. Am J Med Sci. 2001;321(4):249-79. http://dx.doi.org/10.1097/00000441200104000-00006. PMid:11307867.

20. Soares KKD, Gomes ELFD, Beani A Jr, Oliveira LVF, Sampaio LMM, Costa D. Avaliação do desempenho físico e funcional respiratório em obesos. Fisioter Mov. 2012;24(4):697-704. http://dx.doi.org/10.1590/S0103-51502011000400014.

21. Sarikaya S, Cimen OB, Gokcay Y, Erdem R. Pulmonary function tests, respiratory muscle strength, and endurance of persons with obesity. Endocrinologist. 2003;13(2):136-41. http://dx.doi.org/10.1097/01.ten.0000076216.95014.97.

22. Costa TR, Lima TP, Gontijo PL, Carvalho HA, Cardoso FPF, Faria OP, et al. Correlação da força muscular respiratória com variáveis antropométricas de mulheres eutróficas e obesas. Rev Assoc Med Bras. 2010;56(4):403-8. http://dx.doi. org/10.1590/S0104-42302010000400011. PMid:20835635.

23. D'Ávila Melo SM, Melo VA, Menezes RS Fo, Santos FA. Efeitos do aumento progressivo do peso corporal na função pulmonar em seis grupos de índice de massa corpórea. Rev Assoc Med Bras. 2011;57(5):509-15. http://dx.doi. org/10.1590/S0104-42302011000500007. PMid:22012283.

24. Hickey MS, Carey JO, Azevedo JL, Houmard JA, Pories WJ, Israel RG et al. Skeletal muscle fiber composition is related to adiposity and in vitro glucose transportrate in humans. Am J Physiol. 1995;268(3):453-7. http://dx.doi.org/10.1152/ ajpendo.1995.268.3.E453.

25. Tanner CJ, Barakat HA, Dohm GL, Pories WJ, MacDonald $K G$, Cunningham PR, et al. Muscle fiber type is associated with obesity and weight loss. Am J Physiol Endocrinol Metab. 2002;282(6):E1191-6. http://dx.doi.org/10.1152/ ajpendo.00416.2001. PMid:12006347.

26. Magnani KL, Cataneo AJM. Respiratory muscle strength in obese individuals and influence of upperbody fat distribution. Sao Paulo Med J. 2007;125(4):215-9. http://dx.doi.org/10.1590/S1516-31802007000400004. PMid:17992391.

27. Sahebjami H, Gartside PS. Pulmonary function in obese subjects with a normal FEV1 / FVC ratio. Chest. 1996;110(6):1425-9. http://dx.doi.org/10.1378/ chest.110.6.1425. PMid:8989055.

28. Koenig SM. Pulmonary complications of obesity. AmJ Med Sci. 2001;321(4):249-79. http://dx.doi.org/10.1097/00000441200104000-00006. PMid:11307867.

29. Laghi F, Tobin MJ. Disorders of the respiratory muscles. Am J Respir Crit Care Med. 2003;168(1):10-48. http://dx.doi. org/10.1164/rccm.2206020. PMid:12826594.

30. Poulain M, Doucet $M$, Major GC, Drapeau V, Sériès $F$, Boulet LP, et al. The effect of obesity on chronic respiratory diseases: pathophysiology and therapeutic strategies. CMAJ. 2006;174(9):1293-9. http://dx.doi.org/10.1503/cmaj.051299. PMid:16636330.

31. Ray CS, Sue DY, Bray G, Hansen JE, Wasserman K. Effects of obesity on respiratory function. Am Rev Respir Dis. 1983;128(3):501-6. http://dx.doi.org/10.1164/ arrd.1983.128.3.501. PMid:6614644.

32. Mafort TT, Rufino R, Costa CH, Lopes AJ. Obesity: systemic and pulmonary complications, biochemical abnormalities, and impairment of lung function. Multidiscip Respir Med. 2016;11:28. http://dx.doi.org/10.1186/s40248-016-0066-z. PMid:27408717. 